\title{
An Optimized Kwara State Polytechnic Campus Networks using VLAN
}

\author{
K. Abubakar Sadiq \\ Dept. of Computer Science \\ Kwara State Polytechnic, llorin, \\ Nigeria
}

\author{
J. Kehinde Ayeni \\ Dept. of Computer Science \\ Kwara State Polytechnic, Ilorin, \\ Nigeria
}

\author{
F. Samson Oyedepo \\ Dept. of Computer Science \\ Kwara State Polytechnic, Ilorin, \\ Nigeria
}

\begin{abstract}
The goal of any network infrastructure is high Throughput with minimum delay; all these are achievable by avoiding network traffic congestion. Most network infrastructures use switches to break these bearers of network traffic congestion and collision domain to achieve better Throughput. However, the method is not efficient with an extensive scale network, i.e., wide area network WAN. This paper adopts a virtual local area network (VLAN) that helps to improve Ethernet scalability and segment the network into separate broadcast domains, thereby improving network performance, Throughput, bandwidth utilization, delay, and security by filtering unauthorized groups from accessing resources in another group. The performance of VLAN deployments in the administrative block of Kwara state polytechnic is simulated using Riverbed Modeler Academic Edition. In the investigation, performance metrics like; packet delay, bandwidth utilization, Throughput, traffic sent, and traffic received are compared with a conventional case of no VLAN deployment. The simulation results show that VLAN performs better in all the matrices tested.
\end{abstract}

\section{Keywords}

VLAN, LAN, WAN, Campus Network

\section{INTRODUCTION}

The traditional local area network (LAN) is characterized by a collision domain problem, which occurs when two or more devices connected by hubs sent packets at the same time, causing traffic jams or collision, which leads to loss of packets. After some time, the initial packets are resent when the collision has been resolved, thereby wasting significant time and bandwidth utilization [1]. Different methods were introduced in the past to address collision in LAN, such as the use of switches and bridges, to break the collision domain [2]. However, this method is ineffectual with large scale networks like wide area network (WAN). In this paper, the VLAN proposes to mitigate the collision domain in a large campus network such as Kwara State Polytechnic. The VLAN allows LAN to be segmented into different broadcast domains, reducing the complexity of the network architecture and providing different broadcast domains for different packets. This method reduces the chance of a collision occurring and improves overall network performance, such as Throughput, bandwidth utilization, delay, and better security. The Kwara State Polytechnic administrative block is considered in this work and divided into three broadcast domains, namely, Registry server, Bursary server, and Examination and Records server. The design architecture permits workstations or users not physically located within the group to still make use of the group resources without any hindrance. The rest of this paper is as follows: Section 2 presents a review of past work, Section 3 presents the experimental setup, Section 4 views the results and analysis Section 5 and 6 present the conclusion and references respectively.

\section{REVIEW OF PAST WORKS}

The work in [3] discusses campus network topology design, internet protocol (IP) address configuration, packet traffic in LAN, and VLAN use to separate the traffic generated by different network groups or departments. [4] demonstrated VLAN communication experimentation in both hardware and software aspects. The analysis was made for several involved factors that exist in the experimental teaching mode of the research. [5] shows how three university campuses and one department use VLAN to achieve better network, i.e., achieve access control policies. The research further states VLAN may lead to complexity in the configuration of network devices.

\section{EXPERIMENTAL SETUP}

The simulation is carried out using Riverbed Modeler Academic Edition. The Kwara Polytechnic administrative block comprises the first and second floors, while the first floor is sub-divided into the left and right floor, as shown in Figure 1.

1. The VLAN First Floor Right: accommodates the Deputy Rector administration workstation, Registrar office workstation, Auditors office workstation, a remote staff from the Bursary Department, and lastly, Registry mail server of Kwara State Polytechnic. Also, no member of a group can access another group server, even although they share the same physical location, i.e., the Audit workstation won't be able to access the Deputy Rector workstation.

2. The VLAN First Floor Left: This floor accommodates the Deputy Rector Academic workstation, remote Registry staff, and Bursary remote staff, and Examination and records server of Kwara State Polytechnic.

3. The Second Floor VLAN: This floor accommodates the Rectors office workstation, Bursar office workstation, the Bursary Email server, Registry file transfer protocol (FTP) server, and Bursary FTP server. 


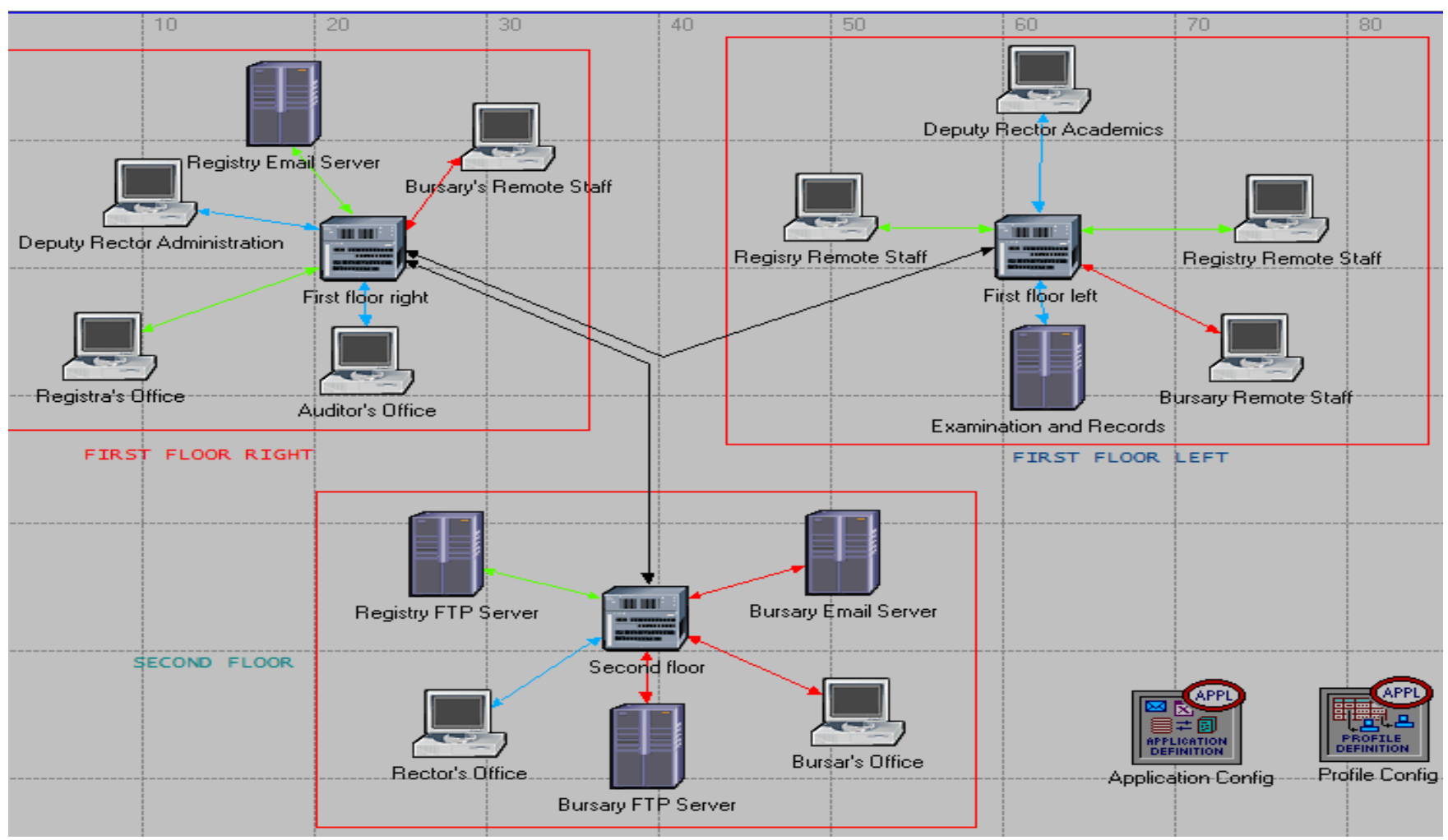

Fig 1: Architectural setup of VLAN Kwara State Polytechnic Administrative Block

\section{RESULT ANALYSIS}

\subsection{Point-to-point Utilization}

The point-to-point utilization is the total work handled by the central processing unit CPU. It also used to measure the general performance of the network. The duration of processing a task and the complexity of the network topology mostly determines the CPU utilization. Figure 2 below shows that the VLAN setup of Kwara State Polytechnic will perform better than the conventional LAN networks.

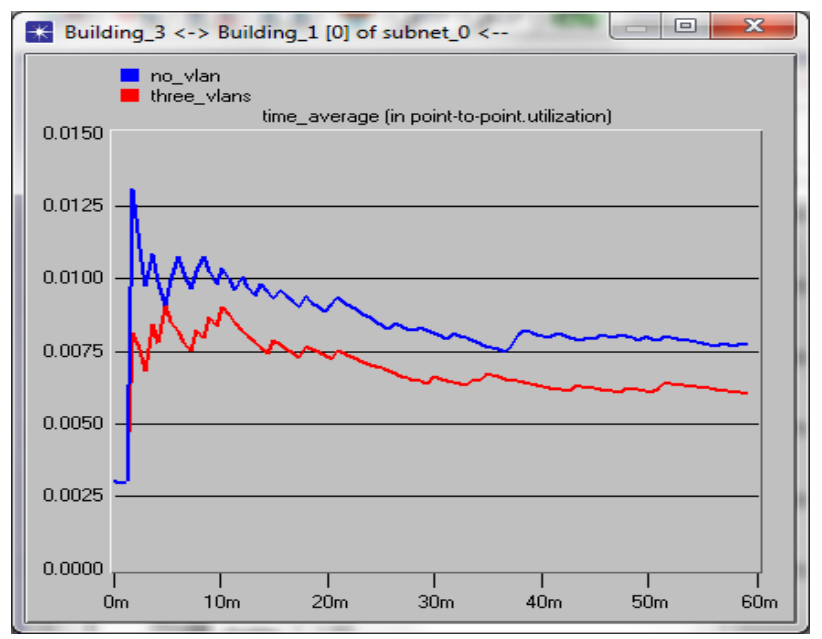

Fig 2: Traffic point-to-point utilization in Bit/Secs

\subsection{Point-to-point Throughput}

The point-to-point Throughput is the rate of successful packets delivered over a communication channel at a given time. The simulation results show VLAN throughput is better than that of convention LAN at a given time of packet transfer.

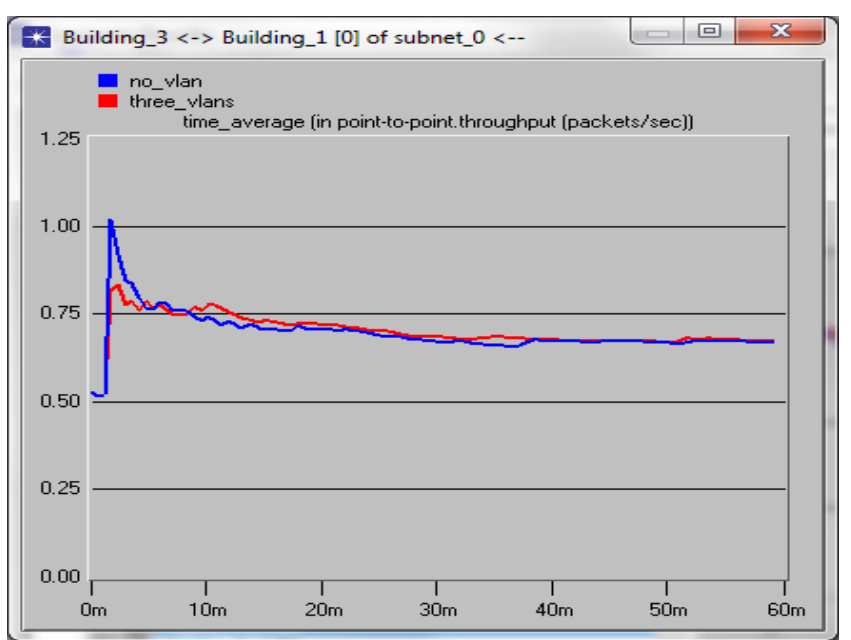

Fig 3: Traffic point-to-point Throughput in Bit/Secs

\subsection{Point-to-point delay}

The time it takes a packet to travel from one end-point to another is referred to as network delay. The simulation shows that VLAN has a lower delay compared to the conventional LAN. 


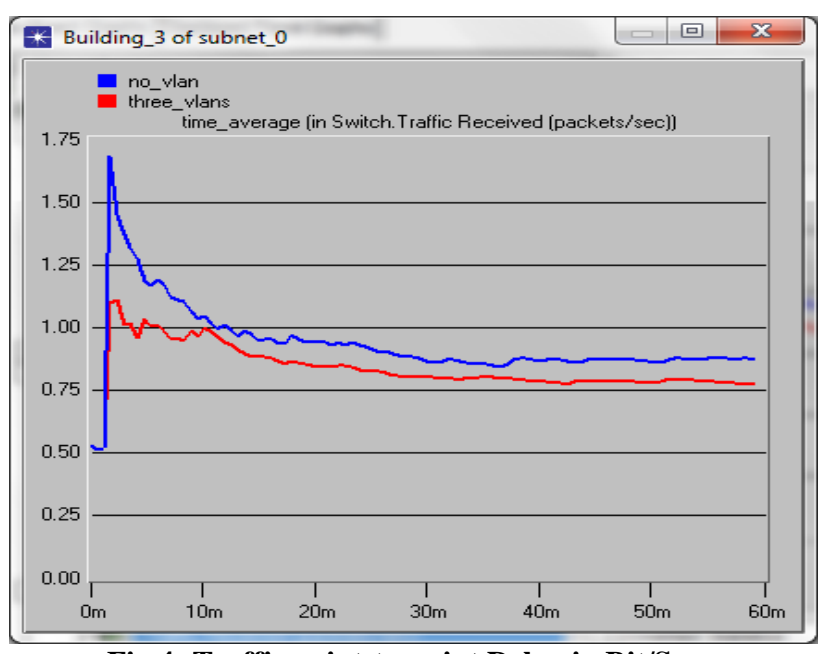

Fig 4: Traffic point-to-point Delay in Bit/Secs

\subsection{Packet Received}

Packet received is the time it takes for a packet to receive from the source. The simulation shows that it takes a longer time for packets to be received from its source in LAN than VLAN.

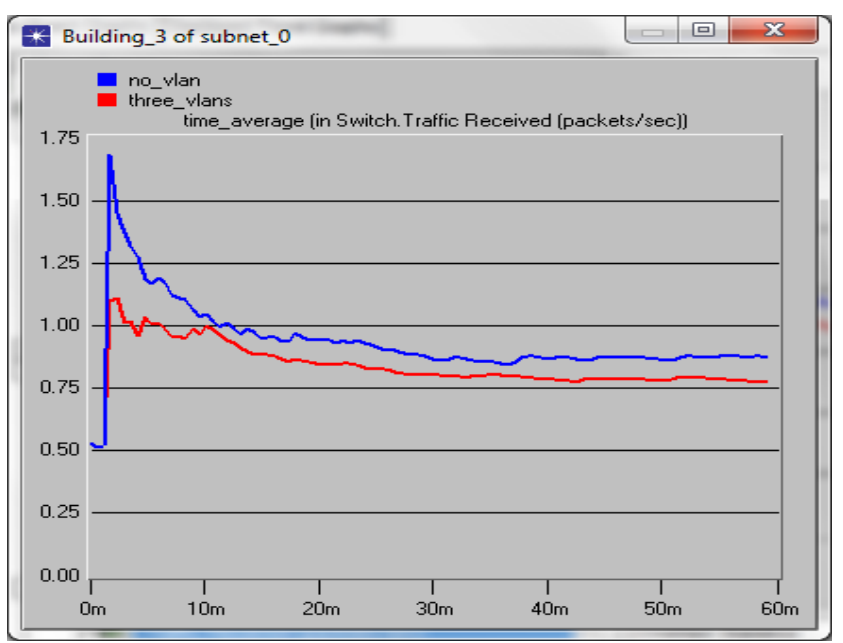

Fig 5: Packet Received in Bit/Secs

\subsection{Packet Forwarded}

Packet sent is the time it takes for a packet to send from the source to the destination. The simulation shows that it takes a longer time for packets to get to its destination in LAN than VLAN.

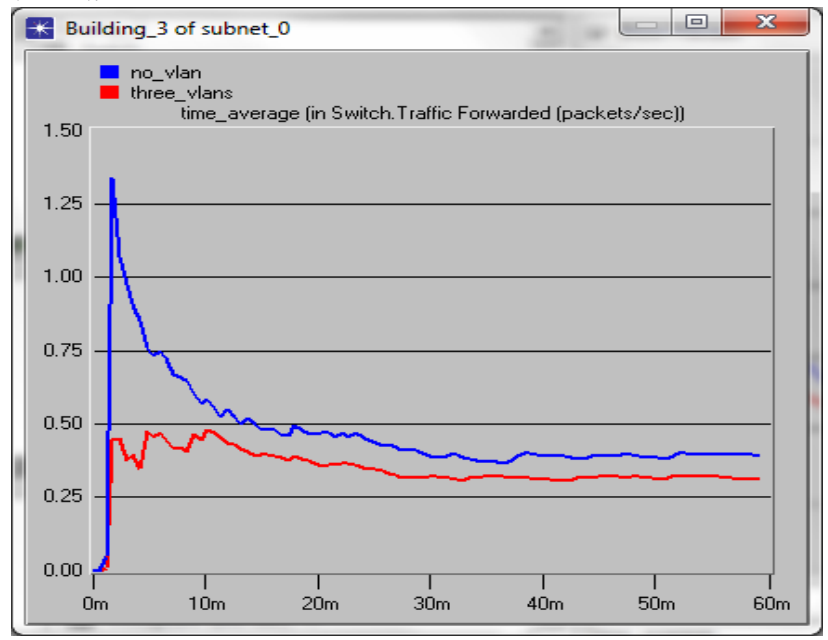

Fig 6: Packet Sent in Bit/Secs

\section{CONCLUSION}

In this paper, two network deployments have been considered using Riverbed modeler academic edition: one with VLAN and the others no VLAN. In the two network deployments, the utilization, Throughput, delay, packet received, and packet sent has been inspected. A comparison among the performance matrices was also carried out. The results show that VLAN will perform better in Kwara State Polytechnic administrative block compared with the present LAN setup.

\section{REFERENCES}

[1] Tambe, S. S., 2015. Understanding Virtual Local Area Networks. International Journal of Engineering Trends and Technology, 25(4), 174-176. https://doi.org/10.14445/22315381/ijett-v25p232

[2] Sadiq, K. A., Tosho Abdullahi, A., \& Bolaji Nasir, S. (2013). Performance Analysis of Ethernet LAN Network Connection under Different Network Devices. International Journal of Information and Network Security (IJINS), 2(6), 462-470. https://doi.org/10.11591/ijins.v2i6.5017

[3] Hossain, M. A., \& Zannat, M. 2019. Simulation and Design of University Area Network Scenario(UANS) using Cisco Packet Tracer. Global Journal of Computer Science and Technology, 19(3), 7-11. https://doi.org/10.34257/gjestgvol19is3pg7

[4] Zhai, G., Long, Z., Zhong, J., \& Cui, Y. (2012). Design and Research of VLAN Communication Experiment Based on the WEB Environment. Procedia Engineering, 29, 2485-2490. https://doi.org/10.1016/j.proeng.2012.01.337

[5] Yu, M., Rexford, J., Sun, X., Rao, S., \& Feamster, N. (2011). A survey of virtual LAN usage in campus networks. IEEE Communications Magazine, 49(7), 98103. https://doi.org/10.1109/mcom.2011.5936161

[6] Cisco , 2016, "Cisco Networking Academy's Introduction to VLANs", Cisco Press, 2014. [Online]. Available: http://www.ciscopress.com. Accessed: Oct. 23.

[7] Daryabar F., Dehghantanha, A., Norouzi, F. and Mahmoodi, F. , 2011, "Analysis of virtual honeynet and VLAN-based virtual networks". International Symposium on Humanities, Science and Engineering Research. IEEE. pp. 73-77.

[8] Kiravuo T., Sarela, M. and Manner, J. , 2013 ,"A survey of ethernet lan security". IEEE Communications Surveys \& Tutorials, Vol. 15, No. 3, pp.1477-1491

[9] D. A. J. AL-Khaffaf, "Improving LAN Performance Based on IEEE802.1Q VLAN Switching Techniques", J. Univ. Babylon, vol. 26, no. 1, pp. 286-297, 2018.

[10] M. Yu, J. Rexford, X. Sun, S. Rao and N. Feamster, "A survey of virtual LAN usage in campus networks", IEEE Commun. Mag., vol. 49, no. 7, pp. 98-103, Jul. 2011.

[11] S. Somasundaram and M. Chandran, A Simulation based study on Network Architecture Using Inter-VLAN Routing and Secure Campus Area Network (CAN), vol. 6, no. 3, pp. 111-121, Mar. 2018.

[12] Giovanni and N. Surantha, "Design and Evaluation of Enterprise Network with Converged Services", Procedia Comput. Sci., vol. 135, pp. 526-533, Jan. 2018. 